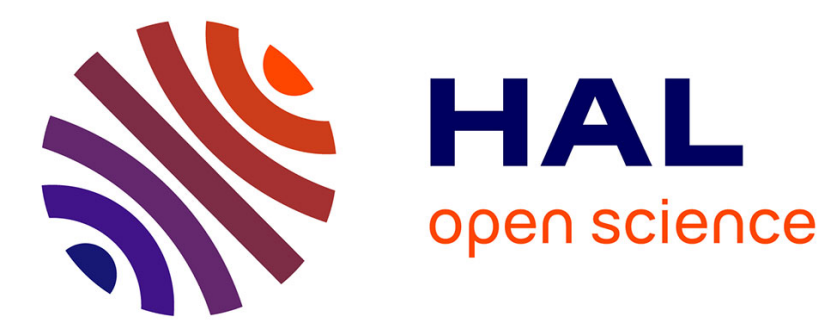

\title{
The extent of the glass transition from molecular simulation revealing an overcrank effect.
} Francois Godey, Alexandre Fleury, Aziz Ghoufi, Armand Soldera

\section{To cite this version:}

Francois Godey, Alexandre Fleury, Aziz Ghoufi, Armand Soldera. The extent of the glass transition from molecular simulation revealing an overcrank effect.. Journal of Computational Chemistry, 2018, 39 (5), pp.255-261. 10.1002/jcc.25069 . hal-01685256

\section{HAL Id: hal-01685256 \\ https://hal-univ-rennes1.archives-ouvertes.fr/hal-01685256}

Submitted on 31 Jan 2018

HAL is a multi-disciplinary open access archive for the deposit and dissemination of scientific research documents, whether they are published or not. The documents may come from teaching and research institutions in France or abroad, or from public or private research centers.
L'archive ouverte pluridisciplinaire HAL, est destinée au dépôt et à la diffusion de documents scientifiques de niveau recherche, publiés ou non, émanant des établissements d'enseignement et de recherche français ou étrangers, des laboratoires publics ou privés. 


\title{
The Extent of the Glass Transition from Molecular Simulation Revealing an Overcrank Effect
}

\author{
François Godey, ${ }^{[a]}$ Alexandre Fleury, ${ }^{[a]}$ Aziz Ghoufi ${ }^{[b]}$ and Armand Soldera ${ }^{[a]}$
}

A deep understanding of the transition between rubber and amorphous state characterized by a glass transition temperature, $T_{\mathrm{g}}$, is still a source of discussions. In this work, we highlight the role of molecular simulation in revealing explicitly this temperature dependent behavior. By reporting the specific volume, the thermal expansion coefficient and the heat capacity versus the temperature, we actually show that the glass transition domain extends to a greater range of temperature, compared with experiments. This significant enlargement

\section{Introduction}

In 1995, P.W. Anderson wrote: “The deepest and most interesting unsolved problem in solid state theory is probably the nature of glass and the glass transition." ${ }^{[1]}$ Despite several years of research, this long-standing problem has not been fully characterized. It is still fascinating that, by decreasing the temperature, properties of polymers such as Young's modulus or viscosity, can change drastically without involving great modification of the overall structure: the polymer remains isotropic. This change in the polymer properties occurs at the glass transition temperature, $T_{\mathrm{g}}$, going from a rubbery state to an amorphous solid, a glass, by decreasing the temperature. A great effort involving theoreticians, experimentalists, and molecular modeling scientists have been devoted to clarify this astonishing phenomenon. ${ }^{[2,3]}$ Molecular simulation offers a particular advantage since it can probe the matter at the atomistic level. Nevertheless, questions remain about the validity of this technique to address this issue, especially about the phase space explored. In this article, we show that molecular simulation can capture the degrees of freedom freeze efficiently, through the time-lapse technique. Motions occurring during the glass transition are, thus, captured in a very different time referential.

Since the seminal study of Roe and Rigby on polyethylene $(\mathrm{PE})_{,}{ }^{[4]}$ uncovering for the first time that $T_{\mathrm{g}}$ can be extracted from atomistic simulation, a significant number of studies have been dedicated to reveal it in other polymers. ${ }^{[5-8]}$ Usually, the determination of $T_{\mathrm{g}}$ is to carry out a simulated dilatometry where the specific volume is reported with respect to the temperature. ${ }^{[4]} \mathrm{A}$ rupture in the linear behavior of the specific volume is an indicator of a change in the properties between two amorphous states, and leads to a value of $T_{\mathrm{g}} \cdot{ }^{[9]}$ Due to correlation with experimental observations, analysis of the molecular reasons that give rise to this tricky transition were, thus, carried out and stimulating conclusions have been extracted. ${ }^{[8,10-13]}$ Nevertheless, it is surprising that a huge width is due to the fast cooling rate, and actually explains the difficulty to locate $T_{\mathrm{g}}$. This result is the manifestation of an overcranking effect used by high-speed cameras to reveal slow-motion. Accordingly, atomistic simulation offers the significant opportunity to show that the transition from the rubber state to the glass phase should be detailed in terms of the degrees of freedom freeze. (c) 2017 Wiley Periodicals, Inc.

difference in the cooling rate between simulation and experiment in order of $10^{[11]}$ times, $^{[14]}$ molecular phenomena accountable for the occurrence of this transition can be grasped. We propose to shed some light on this issue by unveiling some implicit disparities brought by the behavior of thermal properties all along this simulated process. ${ }^{[15]}$ The relevance of our interpretation becomes implicit when such simulation is envisioned as an overcranking effect used in cinematography. This process is carried out by high-speed cameras to reveal motions that cannot be detected otherwise. ${ }^{[16]}$ It, thus, paves the way for further detailed analyses unveiling the freezing of some degrees of freedom.

Glass transition remains an unsolved problem, mainly due to the difficulty to unveil phenomena that occur over such a huge domain of time: from nanoseconds to years (ageing). ${ }^{[2]}$ It, thus, appears challenging to use molecular simulation to capture the physical nature and the microscopic phenomena governing the origin of the glass transition. Due to the very small integration step ( $1 \mathrm{fs}$ ) needed to integrate effectively the equation of motion, the Rouse regime cannot be captured easily, raising the challenges for an efficient exploration of the phase space. The cooling rate is in order of $10^{[11]}$ times more rapid than the experimental one. We addressed the issue differently by setting simulated systems in conditions that approach experiments. Eight simulated systems were initially

[a] F. Godey, A. Fleury, A. Soldera

Centre Québécois sur les Matériaux Fonctionnels (CQMF), Department of

Chemistry, Université de Sherbrooke, Sherbrooke, Québec, Canada, J1K 2R1

[b] A. Ghoufi

Institut de Physique de Rennes, IPR, CNRS-Universite de Rennes 1, UMR CNRS 6251, 35042 Rennes, France

E-mail: armand.soldera@usherbrooke.ca

Contract grant sponsor: Canadian Foundation Innovation (CFI; Calcul Québec and Compute Canada); Contract grant sponsors: Université de Sherbrooke, the Fonds Québécois de la Recherche sur la Nature et les Technologies (FRQNT), and the Natural Sciences and Engineering Research Council of Canada (NSERC) 
set under mechanical equilibrium where hydrostatic pressure equals the stress imposed by the simulated conditions. ${ }^{[17]}$ In fact, the systems should be in thermodynamic equilibrium. ${ }^{[18]}$ It means that the system is simultaneously in mechanical, chemical, radiative, and thermal equilibria. Chemical and radiative equilibria are implicitly attained. The latter one is reached through the use of efficient thermostat algorithm. ${ }^{[19]}$ Reproducibility in mechanical properties, values of $T_{\mathrm{g}}$ and the linear relationship between experimental and simulated $T_{g}$, are some of valuable arguments endorsing the relevance of our approach. ${ }^{[14]}$ However, the question about the very significance of $T_{\mathrm{g}}$ stemming from all-atom simulation remains. Once it is defined properly, the relevance of further studies dedicated to disclose its molecular origin, is perfectly suitable. For this purpose, a scan of this glass transition was undertaken probing evolutions of the thermal expansion coefficient and the heat capacity. Due to very high cooling rate at which these properties are computed, a parallel is done with highspeed cameras. We will, thus, show that differences between simulation and experimental behavior are relevant to enlighten the occurrence of this transition from a molecular simulation perspective. The major difference is the important extent along which the glass transition occurs comparatively to the experimental one. By addressing this concern, we argue that such simulation is an effective tool to grasp some mechanisms at the atomistic level, thus, assisting theories and experiments and simulations at other levels, acting as a time-lapse technique. For this purpose, we focused our studies on a wellstudied polymer due to its simple architecture, polyethylene $(\mathrm{PE})$, and a standard polymer, polystyrene (PS).

\section{Method}

\section{Computational method}

Selection of the initial configurations and their relaxation process are crucial to adequately describe the studied system. A cell with periodic boundary conditions is constituted by four chains of 100 and 125 monomers long for PE and PS, respectively. The generation of the chains embedded in the cell was done through the Self-Avoiding Walk procedure of TheodorouSuter $^{[20]}$ and Meirovitch ${ }^{[21]}$ scanning methods, implemented in the Amorphous_Cell ${ }^{\odot}$ code, in the Materials Studio environment. ${ }^{[22]}$ Fifty configurations were, thus, first obtained. A first selection was made by considering their radius of gyration whose value must not be too far from the average value. The second criterion consists in selecting the eight final configurations exhibiting the lowest energy. A heating-cooling process was then employed to eliminate any endemic stress. Molecular dynamics (MD) in the NPT statistical ensemble where N, P, and T represent the number of molecules, pressure, and temperature, and are kept constant during the simulation. The integration of Newton's equations of motion was performed using the velocity Verlet integration algorithm with a $1 \mathrm{fs}$ integration time step. ${ }^{[23]}$ The Nosé-Hoover thermostat and barostat algorithms were used to maintain constant temperature and pressure, respectively. ${ }^{[19,24]}$ Interactions between atoms were described with the pcff force field. ${ }^{[25]}$ Moreover, the nonbonded interactions have been computed using the Ewald summation, to take into account long range interactions. ${ }^{[26-28]}$ All the MD simulations have been carried out using the LAMMPS code. ${ }^{[29]}$ The heating-cooling process consists in a fast heating process (50 K/200 ps) followed by a lower cooling rate $(20 \mathrm{~K} / \mathrm{ns})$. It has been shown that to get reproducible values of $T_{\mathrm{g}}$, the initial configuration must be in mechanical equilibrium, a "quasi-static" equilibrium state where the stress in the cell balances the internal pressure. ${ }^{[23,30]}$ A uniform hydrostatic compression is imposed to the system until the internal energy reaches a minimum. The configuration at the bottom of the well of energy exhibits an internal pressure that equals the stress imposed by the simulation cell; the mechanical equilibrium is, thus, attained. MD of 5 ns are then run at each temperature, and a configuration is saved at each $500 \mathrm{fs}$. During these dynamics, the trajectory is used to compute averaged properties. More details and the use of this method are available in previous articles. ${ }^{[7,31,32]}$ The specific volume is reported with respect to the temperature, for each ensuing configuration. This simulated dilatometry leads to the value of $T_{\mathrm{g}}$.

\section{Activation energy}

For the calculation of the activation energy, $E_{\mathrm{a}}$, the number of transitions between rotameric states during a period of time at each temperature is first counted. A rotameric state corresponds to a dihedral angle associated with a bond that is in a local minimum energy state. ${ }^{[33]}$ Typical examples are the three rotameric states defined for a $\mathrm{C}-\mathrm{C}$ bond along the polyethylene (PE) chain: trans $(t)$, gauche plus $(g+)$, and gauche minus $(\mathrm{g}$-) states corresponding to dihedral angles of 0 deg., 120 deg., and -120 deg., respectively. The transition between the minima of energies must be defined from an MD perspective. $^{[34]}$ Hotston et al. proposed to count transitions included in a window of 40 deg. width which is centered on one of the three rotameric states $\left(t, g+\right.$, and $g$-) ${ }^{[35]}$ Sumpter et al. consider the occurrence of a dihedral jump when it is greater than 90 deg. ${ }^{[20]}$ Moreover, Wu made a distinction between two types of jumps (transitions), a "shallow jump" and a "deep

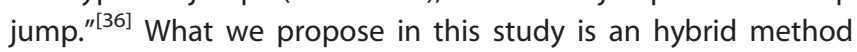
between the two latter methods. We actually introduced a lag time (the time interval needed for a trajectory to lose memory of how the system entered a state) usually ranges from 0.1 to $1 \mathrm{ps}^{[37]}$ A typical example for such a transition between two rotameric states is shown in Figure 1. To reduce the inherent noise of the dihedral angle, we smoothed the data using a sliding average. This average is displayed in Figure 1 with blue circle dots. We also considered a series of conditions. These conditions are as follows: (1) during a transition, the difference between the two involved dihedral angles must be greater than 40 deg. We, thus, used all dihedral angles, and this threshold of $40 \mathrm{deg}$. Allows for a consideration of "shallow jumps" described by $\mathrm{Wu}$; (2) the torsion angle of the new rotameric state must exist for more than 1.5 ps to avoid counting abrupt changes of states. 


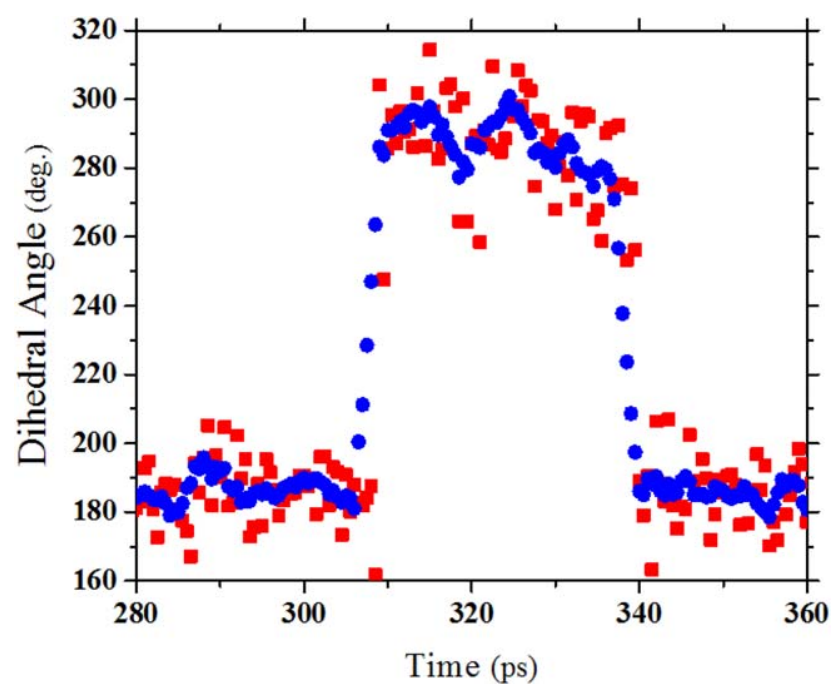

Figure 1. Backbone dihedral angle captured during an MD simulation ( at $180 \mathrm{~K}$, and the corresponding sliding average (O). [Color figure can be viewed at wileyonlinelibrary.com]

Once conformational states and transitions have been defined, the transition rate between these states can be computed for all the backbone bonds at different temperatures, establishing an Arrhenius plot.

$$
v(T)=A \exp \left(-\frac{E_{\mathrm{a}}}{R T}\right)
$$

Where $v(T)$, the rate of conformational transitions, corresponds to the number of transitions divided by the time during which these transitions are happening, at a temperature $T$. The pre-exponential factor $A$ is considered constant and is not discussed in this article. $E_{\mathrm{a}}$, and $R$ are the activation energy $(\mathrm{kcal} / \mathrm{mol})$ of conformational transition, and the ideal gas constant $(\mathrm{kcal} /(\mathrm{mol} . K))$, respectively. $E_{\mathrm{a}}$ is directly deduced from the slope of the graph of $\ln [v(T)]$ with respect to the inverse of the temperature, at temperatures above $T_{\mathrm{g}} \cdot{ }^{[36,38,39]}$

\section{Results and Discussion}

\section{Dilatometric results}

The method generally employed to extract $T_{\mathrm{g}}$ from molecular simulation is the simulated dilatometry. ${ }^{[4]}$ It consists in reporting the specific volume at each temperature, as shown in Figure 2 for pure PE. The actual display of a discontinuity in the linear behavior of the specific volume, as the system is cooling down, is the sign of a change in the molecular behavior. For $\mathrm{PE}$, a discontinuity is found at $T_{1}=248 \mathrm{~K}$, as displayed in Figure $1 \mathrm{a}$. It can be attributed to $T_{\mathrm{g}}$, indicating that it is higher than the recognized experimental one at $195 \mathrm{~K}^{[4,7,34,40]} \mathrm{How}$ ever, by disclosing a greater domain of temperatures, the localization of $T_{\mathrm{g}}$ becomes hazardous. A second deviation from the linear behavior in the dilatometry spectrum (Fig. 2b) is observed at higher temperature, leading to a second rupture in the slope at $T_{2}=400 \mathrm{~K}$. Experimentally, $T_{\mathrm{g}}$ corresponds to the intersection between the two straight lines drawn at higher and lower temperatures. ${ }^{[41,42]}$ By applying this procedure as shown in Figure $2 \mathrm{~b}, T_{g}^{d}$ of $300 \mathrm{~K}$ is deduced; the superscript $d$ stands for dilatometry. It is clearly higher than the experimental one but can be related to the experimental value through the Williams-Landel-Ferry equation. ${ }^{[14]}$ The existence of two temperatures stemming from ruptures in the slope of the specific volume leads to defining three domains of different behavior. Domain I is defined for temperatures below $T_{1}$, that is, the glass domain; domain II for temperatures between $T_{1}$ and $T_{2}$, where $T_{g}^{d}$ is supposed to be located; domain III for temperatures higher than $T_{2}$, that is, in the amorphous or rubber state. Domain of the glass transition, domain II, is much broader in temperature, with $\Delta T=T_{2}-T_{1}=152 \mathrm{~K}$, than the experimental one, typically spread over 3-5 K. ${ }^{[15]}$ This important extent must be definitively characterized, and first confirmed with another polymer, PS.

The occurrence of three domains of temperatures in the simulated dilatometry graph of PS is confirmed in Figure 3. They are limited by $T_{1}=400 \mathrm{~K}$ and $T_{2}=560 \mathrm{~K}$ leading to $T_{g}^{d}=$ $466 \mathrm{~K}$. This value is clearly higher than the experimental one
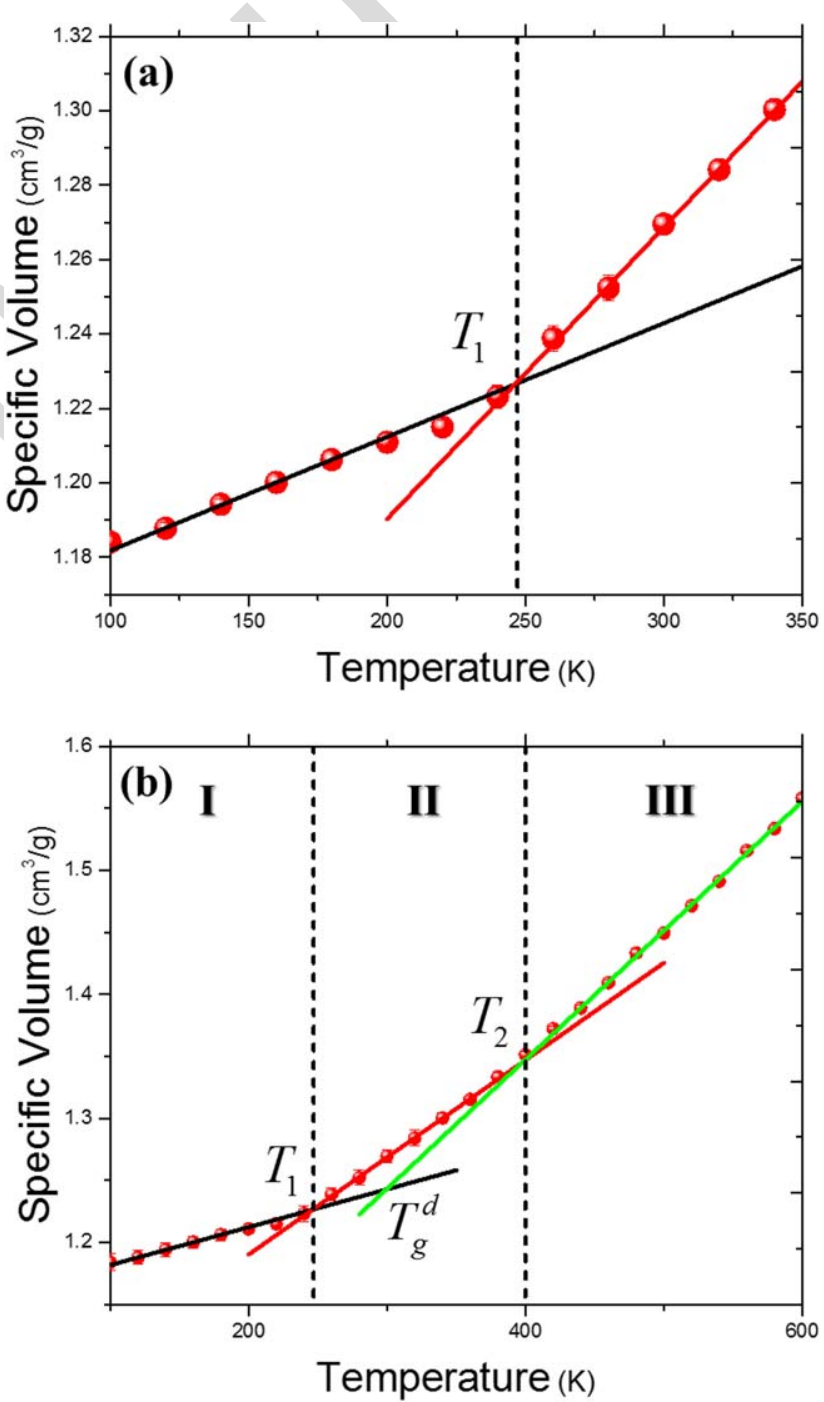

Figure 2. Specific volume with respect to the temperature (K) for PE: a) from 100 to $350 \mathrm{~K}, \mathrm{~b}$ ) and from 100 to $600 \mathrm{~K}$. [Color figure can be viewed at wileyonlinelibrary.com] 


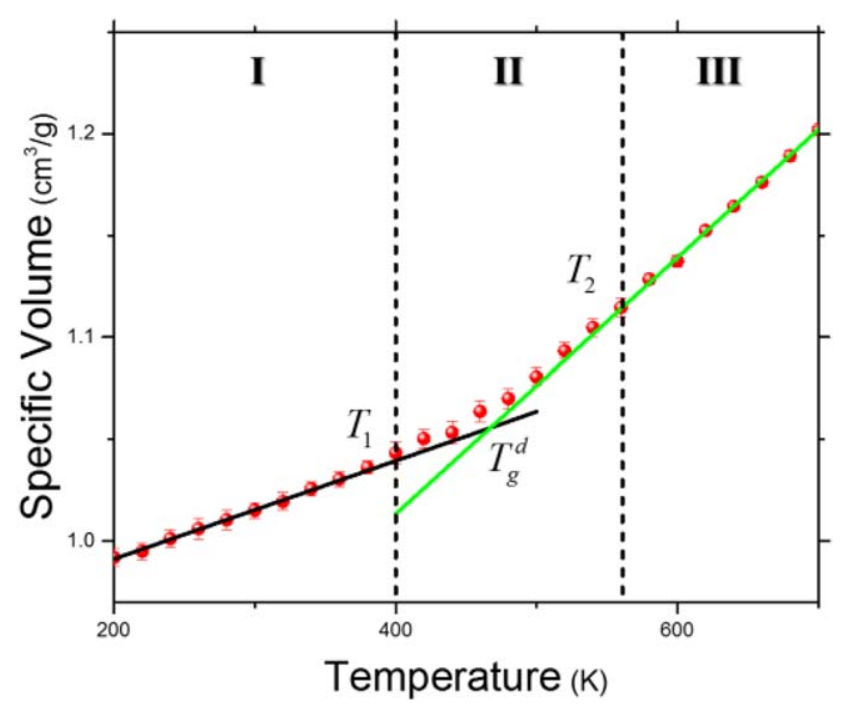

Figure 3. Specific volume with respect to the temperature from 200 to $700 \mathrm{~K}$ of PS. [Color figure can be viewed at wileyonlinelibrary.com]

of $383 \mathrm{~K} \cdot{ }^{[43]}$ The domain of the glass transition range, that is, domain II, remains particularly important with $\Delta T=T_{2}$ $T_{1}=160 \mathrm{~K}$. To identify appropriately this pronounced difference in the thermal transition, further investigation and, thus, comparison with experimental data must be achieved. The behavior of thermal expansion coefficient and heat capacity with respect to the temperature are of particular interest since they can be captured experimentally and by simulation.

\section{Thermal expansion coefficient}

The thermal expansion coefficient, $\alpha$, is defined in eq. (2).

$$
\alpha=\frac{1}{V}\left(\frac{\partial V}{\partial T}\right)
$$

where $V$ is the volume, and $T$ is the temperature in Kelvin. Since the thermodynamic limit is not attained using all-atom simulation, formulae involving fluctuations must be used [eq. (3)].

$$
\bar{\alpha}=\overline{\left(\frac{\langle U V\rangle}{\langle V\rangle}-\langle U\rangle\right)} \frac{1}{k_{\mathrm{B}} T^{2}}
$$

Where $U$ is the internal potential energy, $V$ is the volume of the simulated cell, $k_{\mathrm{B}}$ is the Boltzmann constant, and $T$ is the temperature in Kelvin. The brackets indicate that the average is taken from MD trajectory of each configuration, while the error bar considers the average over the eight configurations. Behaviors of $\alpha$ versus the temperature are shown in Figures 4 and ${ }^{[5]}$ for PE and PS, respectively. In each case, the domains stemming from the simulated dilatometry are displayed. Moreover, a linear fit for low temperature data is shown. It is aimed at revealing the reach of a linear behavior in the glass state as temperature is decreasing.

The domains of temperatures previously defined by the simulated dilatometry correspond to the regions bordered by changes in the behavior of $\alpha$ with temperature (Figs. 4 and 5). By lowering the temperature, $\alpha$ begins to decrease near $T_{2}$ for both polymers. A lessening of the decrease is observed at $T_{1}$ for PS, and slightly below $T_{1}$ for PE. The fluctuation formulae correctly extract the ruptures in the slope observed in the simulated dilatometry graph, and, thus, the ensuing domains. A difference must however be noted for PE at low temperatures, that is, slightly below $T_{1}$.

The actual glass transition domain stemming from simulation remains large comparatively to the experimental one. However, despite this significant difference, there is a strong agreement between simulated and experimental values of $\alpha$ in the glass and liquid, respectively $\alpha_{g}$ and $\alpha_{1}$, as shown in Table 1 , especially for PS. ${ }^{[44]}$ For PE, experimental values of $\alpha$ depend on the technique used, ${ }^{[44-46]}$ but remain in the same order of magnitude. Simulated values are, thus, in agreement with experimental data. Such a concordance is an additional asset to confirm the importance to set the initial configurations in mechanical equilibrium, depicting efficiently the glass state. Moreover, MD definitively uncovers specific behavior occurring at the glass transition. For a 5 ns step duration, the experimental density cannot be attained, as it is done in the glass state through an imposed compression to get mechanical equilibrium. Nevertheless, the ensuing difference in the thermal expansion coefficient, $\Delta \alpha=\alpha_{1}-\alpha_{g}$ can be used in the Simha-Boyer relation [eq. (4)], ${ }^{[44]}$ enabling the calculation of $T_{g}$, as shown in Table 1.

$$
\Delta \alpha \cdot T_{g}^{\alpha}=\text { constant }
$$

The constant in the Shima-Boyer relation slightly varies according to polymer. It is reported in Table 1 for PE and PS. A value of $T_{g}^{\alpha}$ is then achieved. It is reported in Table 1 for each studied polymer. In the original article of Boyer and Simha, a value of $T_{\mathrm{g}}$ of $143 \mathrm{~K}$ was proposed for PE. Most of the studies about $T_{g}$ of PE give a value of $195 \mathrm{~K}^{[4,7,34]}$ Application of eq. (4) yields values of $T_{\mathrm{g}}$ approaching the experimental one (Table 1).

These results greatly support the relevance in using atomistic simulation to disclose the glass transition phenomenon.

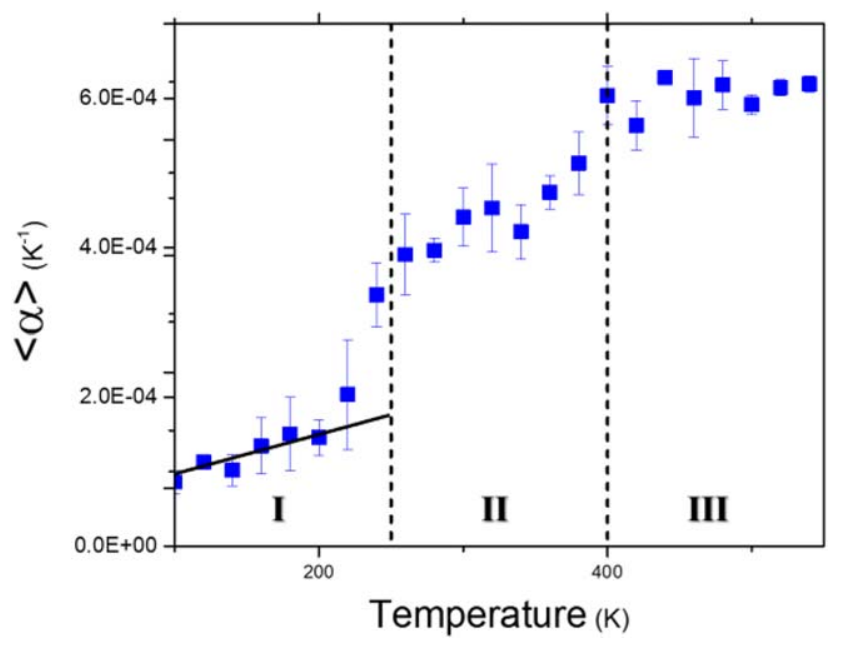

Figure 4. The thermal expansion coefficient of PE versus the temperature from 100 to $500 \mathrm{~K}$, with the simulated dilatometry superimposed. [Color figure can be viewed at wileyonlinelibrary.com] 
Table 1. Experimental and simulated thermal expansion coefficients for PE and PS.

\begin{tabular}{|c|c|c|c|c|}
\hline \multirow[b]{2}{*}{ Property } & \multicolumn{2}{|c|}{ PE } & \multicolumn{2}{|c|}{ PS } \\
\hline & $\operatorname{Exp}^{a}$ & $\mathrm{Sim}^{\mathrm{b}}$ & $\operatorname{Exp}^{a}$ & $\operatorname{Sim}^{b}$ \\
\hline$\alpha_{g}$ & $\begin{array}{l}1.3410^{-4} \mathrm{~K}^{-1}[33] \\
1.4310^{-4} \mathrm{~K}^{-1}[21] \\
2.9510^{-4} \mathrm{~K}^{-1}[22]\end{array}$ & $1.810^{-4} \mathrm{~K}^{-1}$ & $2.510^{-4} \mathrm{~K}^{-1[33]}$ & $2.110^{-4} \mathrm{~K}^{-1}$ \\
\hline$\alpha_{1}$ & $\begin{array}{l}2.710^{-4} \mathrm{~K}^{-1}[41] \\
8.910^{-4} \mathrm{~K}^{-1}\end{array}$ & $6.010^{-4} \mathrm{~K}^{-1}$ & $5.510^{-4} \mathrm{~K}^{-1}[33]$ & $5.010^{-4} \mathrm{~K}^{-1}$ \\
\hline$\Delta \alpha$ & $\begin{array}{l}1.3610^{-4} \mathrm{~K}^{-1}[21] \\
5.9510^{-4} \mathrm{~K}^{-1}[22]\end{array}$ & $4.210^{-4} \mathrm{~K}^{-1}$ & $3.010^{-4} \mathrm{~K}^{-1}$ & $2.910^{-4} \mathrm{~K}^{-1}$ \\
\hline $\begin{array}{l}\Delta \alpha T_{\mathrm{g}} \\
T_{g}^{\alpha}\end{array}$ & $\begin{array}{c}0.097-0.113^{[42]} \\
143 \mathrm{~K}^{[42]}\end{array}$ & $\begin{array}{c}/ \\
230-269 \mathrm{~K}\end{array}$ & $\begin{array}{l}0.112^{[42]} \\
373 \mathrm{~K}^{[42]}\end{array}$ & $\begin{array}{c}/ \\
386 \mathrm{~K}\end{array}$ \\
\hline
\end{tabular}

[a] Experimental. [b] Simulation.

However, despite these encouraging outcomes, the large size of the glass transition domain remains an issue that must be further investigated. The behavior of the heat capacity with temperature is, thus, examined.

\section{Heat capacity}

The heat capacity at constant volume $\left(C_{v}\right)$ is computed according to fluctuations of energy, as shown in eq. (5).

$$
\overline{C_{v}}=\frac{\overline{\left(\left\langle U^{2}\right\rangle-\langle U\rangle^{2}\right)}}{k_{B} T^{2}}
$$

Where $U$ is the internal potential energy, $k_{\mathrm{B}}$ is the Boltzmann constant, and $T$ is the temperature in Kelvin. The brackets indicate that the average is taken from MD trajectory of each configuration, while the bar stands for the average over the eight configurations. $C_{v}$ versus temperature is reported in Figures 6 and 7, for PE and PS, respectively. The three temperature domains defined in the simulated dilatometry are also indicated.

For both polymers, values are slightly lower than experimental data, ${ }^{[47-49]}$ mainly due to the fact that the Einstein contribution is not taken into account. Very interestingly is the behavior of $C_{v}$

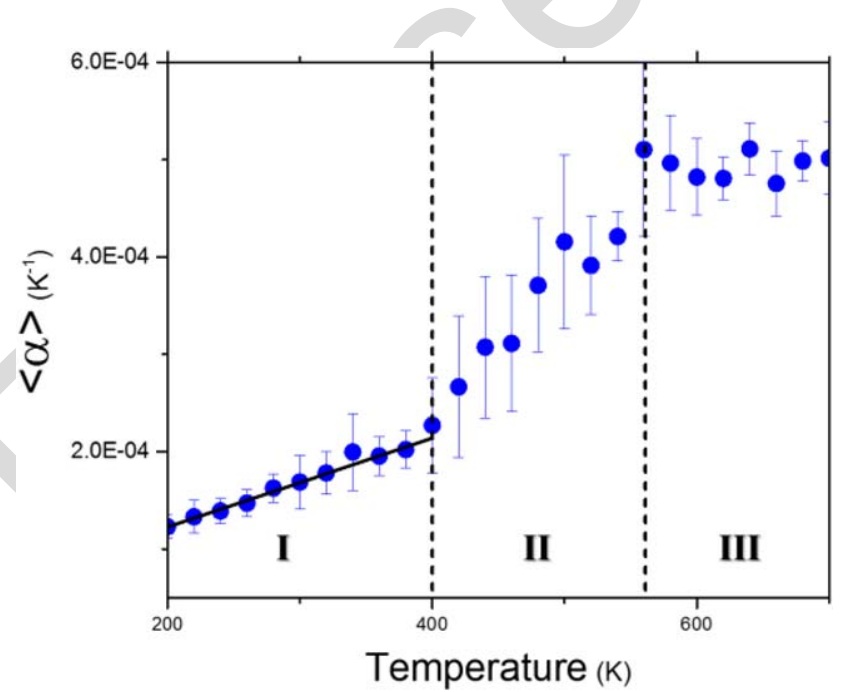

Figure 5. The thermal expansion coefficient of PS versus the temperature: from 200 to $700 \mathrm{~K}$, with the domains revealed by simulated dilatometry. [Color figure can be viewed at wileyonlinelibrary.com] during the glass transition whose shape is in agreement with experimental curve. ${ }^{[15]}$ As the temperature is cooling down, a decrease in $C_{v}$ is normally observed. Considering the error bars, the lessening can be regarded as initiating at $T_{2}$. The linear expected behavior in the glass state is attained at $T_{1}$ for PS (Fig. 6), and slightly below for PE (Fig. 5). This extension in the decrease of $C_{v}$ at temperature below $T_{1}$ was also observed for $\alpha$. It has been actually observed experimentally in $\mathrm{PE}$, and mentioned by Wunderlich, due to trans-gauche exchanges. ${ }^{[15]}$ The actual shape of $C_{v}$ with temperature is in agreement with experimental observation. Accordingly a $T_{g}^{C_{v}}$ is deduced. Due to the imprecision at lower temperatures, $T_{g}^{C_{V}}$ is roughly located in the middle of the two transitions previously defined, $T_{1}$ and $T_{2}$, leading to $T_{g}^{C_{v}} \approx 325 \mathrm{~K}$ and $480 \mathrm{~K}$, for PE and PS, respectively. They are clearly higher than experimental $T_{\mathrm{g}}$.

The broadening of the glass transition domain has been confirmed as witnessed by the thermal behavior of the specific volume, the thermal expansion coefficient, and the heat capacity. Experimentally, the occurrence of crystalline components explains any broadening of $C_{v}$ during the glass transition, but cannot be applied in our wholly amorphous systems. Due to the very high cooling rate comparatively to the experimental one, with a ratio

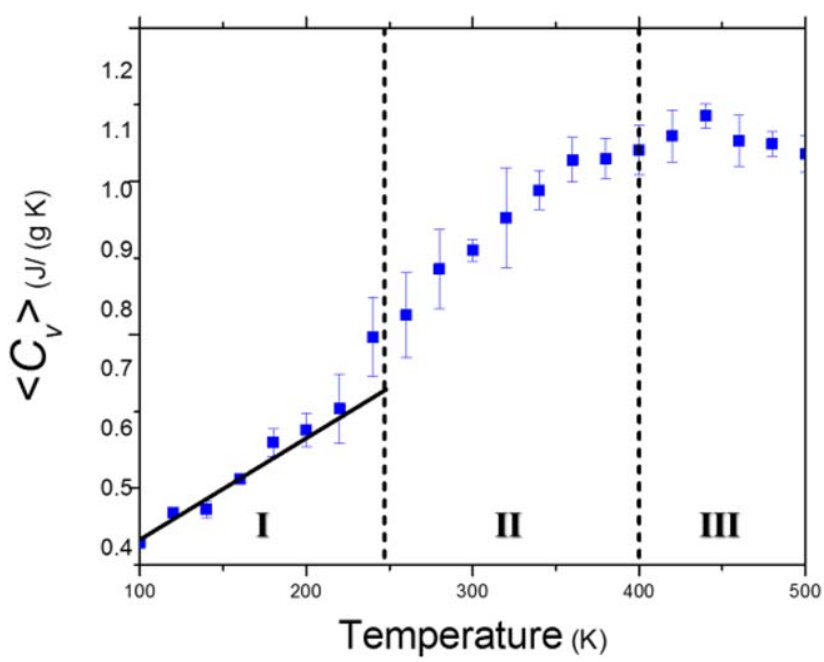

Figure 6 . The heat capacity at constant volume with respect to the temperature (K): from 100 to $600 \mathrm{~K}$ for PE. The three temperature domains defined in the simulated dilatometry can be observed. [Color figure can be viewed at wileyonlinelibrary.com] 


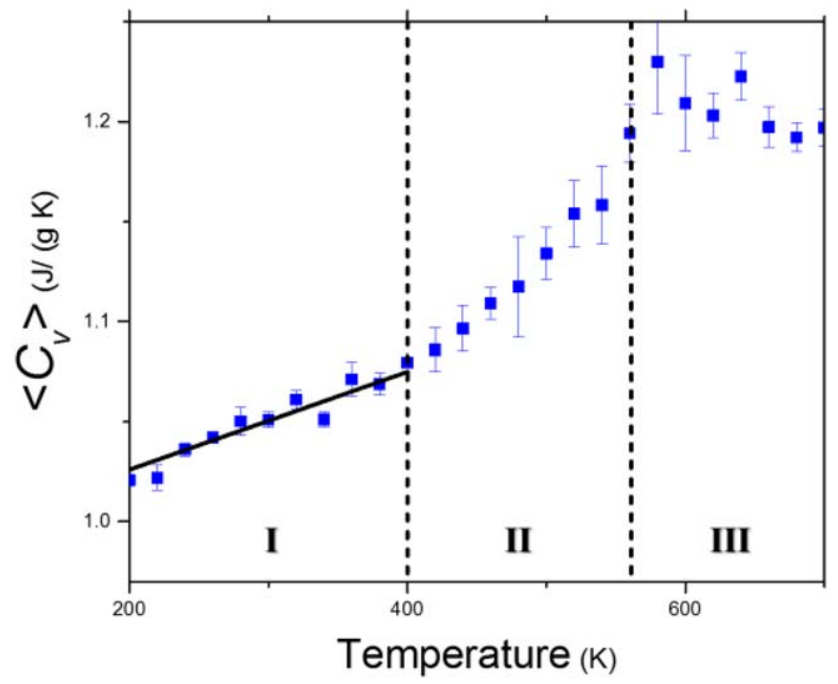

Figure 7. The heat capacity at constant volume with respect to the temperature (K): from 200 to $700 \mathrm{~K}$ for PS. The three temperature domains defined in the simulated dilatometry are shown too. [Color figure can be viewed at wileyonlinelibrary.com]

in the order of $10^{[11]}$ more rapid, all the degrees of freedom that account for the glass transition to occur cannot be frozen immediately. Nevertheless, in the glass state where the mechanical equilibrium was previously achieved, these degrees of freedom, mainly backbone dihedral angles, are frozen. The glass state is, therefore, correctly reproduced. ${ }^{[31,50]}$ Accordingly, the all-atom simulation represents adequately the glass transition, provided that it occurs through a longer range of temperatures. It can, thus, be compared to the result of using of a high-speed camera that captures very rapid motions that are impossible to detect in real time. ${ }^{[51]}$ As a consequence, any properties that are computed in this domain must be averaged over this domain. We consider calculation of the activation energy as a noteworthy example.

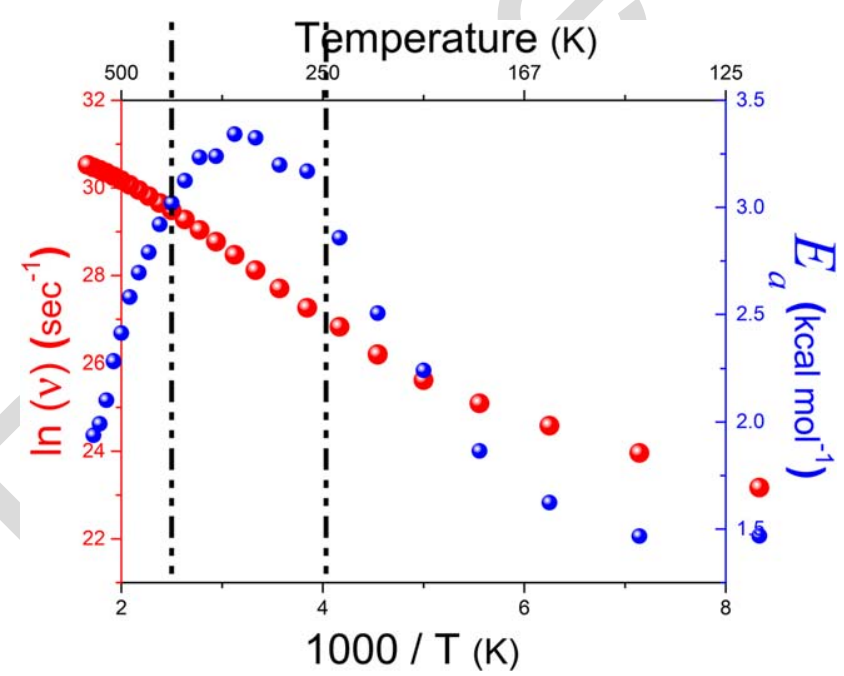

Figure 8. Arrhenius plot where the natural logarithm of the transition per second is reported with respect to the inverse of temperature for PE. $E_{\mathrm{a}}$ stemming from the slope at each point is also reported. The three temperature domains defined in the simulated dilatometry can be observed. [Color figure can be viewed at wileyonlinelibrary.com]

\begin{tabular}{|c|c|c|c|c|}
\hline & \multicolumn{2}{|c|}{ PE } & \multicolumn{2}{|c|}{ PS } \\
\hline & $\operatorname{Sim}^{[a]}$ & $\operatorname{Exp}^{[b]}$ & $\operatorname{Sim}^{[a]}$ & $\operatorname{Exp}^{[b]}$ \\
\hline$T_{a}^{d}(\mathrm{~K})$ & 300 & $195^{[4,7,22]}$ & 466 & $358-383^{[23]}$ \\
\hline$T_{g}^{\alpha}(\mathrm{K})$ & $230-269$ & $143^{[21]}$ & 386 & $373^{[21]}$ \\
\hline$T_{1}(\mathrm{~K})$ & 248 & & 400 & \\
\hline$T_{g}^{C_{v}}(\mathrm{~K})$ & 325 & & 480 & \\
\hline
\end{tabular}

\section{Activation energy}

Conformational transition rates between rotameric states, ${ }^{[33]}$ that is, conformations of local minimum energy, can be computed at different temperatures and then reported in an Arrhenius plot. $^{[52,53]}$ The slope directly yields the activation energy $\left(E_{\mathrm{a}}\right)$, as it is shown in Figure 8 . The value of $E_{\mathrm{a}}$ can then be averaged over the domain of temperature between $T_{1}$ and $T_{2}$, leading to a small discrepancy: $3.20 \pm 0.05 \mathrm{kcal} / \mathrm{mol}$. This value corresponds to the potential energy barrier that needs to rotate around backbone bonds. ${ }^{[37-39]}$ An average value is deduced from the glass transition domain. However, as $C_{v}$ changes all along this region, it reveals that by decreasing the temperature degrees of freedom progressively freeze. As in the overcranking effect, details that cannot be observed from other techniques can now be captured. A representation of the energy landscape can, thus, be envisioned. The unveiling of the degrees of freedom freezing through a slow decline of the heat capacity will be discussed specifically in a forthcoming article.

Another corollary of this overcranking is that the location of $T_{\mathrm{g}}$ extracted from the atomistic simulation is not straightforward. In Table 2, all the different $T_{\mathrm{g}}$ that can be provided by the all-atom simulation are displayed, except $T_{2}$ that corresponds to the beginning of the glass transition.

The value that approaches the most the experimental data is $T_{g}^{\alpha}$ stemming from the application of the Simha-Boyer relation. However, this empirical relationship cannot be used for new polymers. $T_{1}$ is the temperature where number of transitions between rotameric states declines seriously, as confirmed by the Arrhenius plot (Fig. 8). The actual difficulty to locate $T_{\mathrm{g}}$ makes molecular simulation a perfect tool to enlighten molecular changes that occur during the glass transition, by revealing the freezing of the degrees of freedom in a greater domain of temperatures.

\section{Conclusions}

The glass transition is a domain of temperatures where the conversion from the rubbery state to the glass state occurs. Experimentally, for a specific sample, it is defined in a narrow range of temperatures making possible the identification of $T_{\mathrm{g}}$. We showed that in all-atom simulation there are different ways to define it. It is possible to associate one transition temperature as an effective $T_{\mathrm{g}}$ : it can be the temperature at which the rubbery state becomes a glass ( $T_{1}$ in the text), or the intersection between the two slopes at high and low temperatures 
in the simulated dilatometry ( $T_{g}^{d}$ in the text). A specific property must, thus, be averaged to compare efficiently to experimental data. Moreover, we showed that as $C_{v}$ decreases regularly, freeze of degrees of freedom occurs in a long range of temperature, in the order of $150 \mathrm{~K}$. This technique can, thus, be compared with the high-speed camera which reveals motions that are not detectable by human eyesight. The phenomenon is definitively captured, but all the specific motions can now be wholly detailed. Since a constant activation energy is observed that must be correlated with a slow decline of $C_{v \prime}$ it, thus, paves the way for further studies exploring this molecular aspect of the glass transition, and especially by disclosing the energy landscape. ${ }^{[54]}$

Keywords: glass transition - atomistic simulation - overcrank · polymers $\cdot$ molecular dynamics

[1] H. Weintraub, M. Ashburner, P. N. Goodfellow, H. F. Lodish, C. J. Arntzen, P. W. Anderson, T. M. Rice, T. H. Geballe, A. R. Means, Science 1955, 267, 1609.

[2] E. Donth, The Glass Transition; Springer Verlag: New York, 2001.

[3] J. Rault, Physical Aging of Glasses: The VFT Approach; Nova Science Publishers: New York, 2009.

[4] D. Rigby, R.-J. Roe, J. Chem. Phys. 1987, 87, 7285.

[5] R. Wu, B. Kong, X. Yang, Polymer 2009, 50, 3396.

[6] M. Ozmaian, R. Naghdabadi, J. Polym. Sci. Part B Polym. Phys. 2014, $52,907$.

[7] A. Soldera, Mol. Simul. 2012, 38, 762.

[8] J. Han, R. H. Gee, R. H. Boyd, Macromolecules 1994, 27, 7781.

[9] G. J. Moro, J. Chem. Phys. 1991, 94, 8577.

[10] N. Metatla, A. Soldera, Macromolecules 2007, 40, 9680.

[11] M. Bulacu, E. Van Der Giessen, Phys. Rev. E Stat. Nonlin. Soft Matter Phys. 2007, 76, 011807.

[12] J. Baschnagel, M. Fuchs, J. Phys. Condens. Matter 1995, 7, 6761.

[13] R. Wu, X. Qiu, K. Fu, T. Zhang, X. Yang, J. Phys. Chem. B 2015, 119, 9959.

[14] A. Soldera, N. Metatla, Phys. Rev. E 2006, 74, 061803.

[15] B. Wunderlich, Thermal Analysis of Polymeric Materials; Springer: Berlin, 2005.

[16] V. R. Lee, J. Sci. Educ. Technol. 2015, 24, 178.

[17] N. Metatla, A. Soldera, Macromol. Theory Simul. 2011, 20, 266.

[18] F. Porzio, E. Cuierrier, C. Wespiser, S. Tesson, R. Underhill, A. Soldera, Mol. Simul. 2017, 43, 169.

[19] S. Nosé, Mol. Phys. 1984, 52, 255.
[20] D. N. Theodorou, U. M. Suter, Macromolecules 1985, 18, 1467.

[21] H. Meirovitch, J. Chem. Phys. 1983, 79, 502.

[22] Amorphous_Cell. Materials Studio. Accelrys Inc.: San Diego, CA.

[23] J. M. Haile, Molecular Dynamics Simulation: Elementary Methods; Wiley: New York, 1992

[24] W. G. Hoover, Phys. Rev. A 1985, 31, 1695.

[25] J. R. Maple, et al., J. Comput. Chem. 1994, 15, 162

[26] O. Lebel, T. Maris, M.-E. Perron, È. Demers, J. D. Wuest, J. Am. Chem. Soc.2006, 128, 10372.

[27] J. D. Wuest, O. Lebel, Tetrahedron 2009, 65, 7393.

[28] M. P. Allen, D. J. Tildesley, Computer Simulation of Liquids; Clarendon Press: Oxford, 1987.

[29] S. Plimpton, J. Comput. Phys. 1995, 117, 1.

[30] N. Metatla, A. Soldera, Macromol. Theor. Simul. 2011, 20, 266.

[31] N. Metatla, A. Soldera, Macromolecules 2007, 40, 9680.

[32] N. Metatla, A. Soldera, Mol. Simul. 2006, 32, 1187.

[33] P. J. Flory, Statistical Mechanics of Chain Molecules; Hanser Publishers, 1989.

[34] Q. Yang, X. Chen, Z. He, F. Lan, H. Liu, RSC Adv. 2016, 6, 12053.

[35] E. Helfand, Z. R. Wasserman, T. A. Weber, Macromolecules 1980, 13, 526.

[36] R. Wu, X. Zhang, Q. Ji, B. Kong, X. Yang, J. Phys. Chem. B 2009, 113, 9077.

[37] A. Hopfinger, J. Macromol. 1989, 22, 2472.

[38] T. Liang, Y. Yang, D. Guo, X. Yang, J. Chem. Phys. 2000, 112, 2016.

[39] J. F. Mano, M. S. Lanceros, J. Appl. Phys. 2001, 89, 1844.

[40] G. Dlubek, K. Saarinen, H. M. Fretwell, J. Polym. Sci, Part B Polym. Phys. 1998, 36, 1513.

[41] G. T. Davis, R. K. Eby, J. Appl. Phys. 1973, 44, 4274.

[42] S. Saito, T. Nakajima, J. Appl. Polym. Sci. 1959, 2, 93.

[43] J. Brandrup, E. H. Immergut, E. A. Grulke, Polymer Handbook, (4th edition), New York, 1999.

[44] R. Simha, R. F. Bayer, J. Chem. Phys. 1962, 37, 1003.

[45] G. K. White, C. L. Choy, J. Polym. Sci. Polym. Phys. Ed. 1984, 22, 835.

[46] J. P. Crine, Polym. Bull. (Berlin) 1986, 15, 375.

[47] J. E. Mark, In Physical Properties of Polymers Handbook; J. E. Mark, Ed.; A.I.P: Woodbury, NY; 1996.

[48] U. Gaur, B. Wunderlich, Macromolecules 1980, 13, 445

[49] J. Wen, Heat Capacities of Polymers; AIP Press, 1996.

[50] S. Palato, N. Metatla, A. Soldera, Eur. Phys. J. E 2011, 34, 90.

[51] I. Fredricson, S. Drevemo, G. Dalin, G. Hjerten, K. Bjorne, Equine Vet; J. 1980, 12, 54.

[52] S. D. Hotston, D. B. Adolf, K. Karatasos, J. Chem. Phys. 2001, 115, 2359.

[53] R. Boyd, G. Smith, Polymer Dynamics and Relaxation; Cambridge University Press: Cambridge, 2007.

[54] P. G. Debenedetti, F. H. Stillinger, Nature 2001, 410, 259.

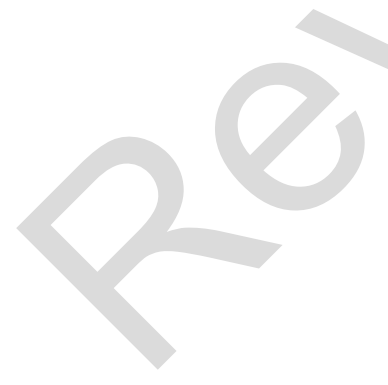

